\title{
Front Matter: Volume 7198
}

, "Front Matter: Volume 7198," Proc. SPIE 7198, High-Power Diode Laser Technology and Applications VII, 719801 (16 March 2009); doi: $10.1117 / 12.824382$

SPIE Event: SPIE LASE: Lasers and Applications in Science and Engineering, SPIE. 2009, San Jose, California, United States 


\section{PROCEEDINGS OF SPIE}

\section{High-Power Diode Laser Technology and Applications VII}

Mark S. Zediker

Editor

26-27 January 2009

San Jose, California, United States

Sponsored and Published by

SPIE

Volume 7198

Proceedings of SPIE, 0277-786X, v. 7198 
The papers included in this volume were part of the technical conference cited on the cover and title page. Papers were selected and subject to review by the editors and conference program committee. Some conference presentations may not be available for publication. The papers published in these proceedings reflect the work and thoughts of the authors and are published herein as submitted. The publisher is not responsible for the validity of the information or for any outcomes resulting from reliance thereon.

Please use the following format to cite material from this book:

Author(s), "Title of Paper," in High-Power Diode Laser Technology and Applications VII, edited by Mark S. Zediker, Proceedings of SPIE Vol. 7198 (SPIE, Bellingham, WA, 2009) Article CID Number.

ISSN 0277-786X

ISBN 9780819474445

Published by

SPIE

P.O. Box 10, Bellingham, Washington 98227-0010 USA

Telephone +1 3606763290 (Pacific Time) · Fax +1 3606471445

SPIE.org

Copyright (C) 2009, Society of Photo-Optical Instrumentation Engineers

Copying of material in this book for internal or personal use, or for the internal or personal use of specific clients, beyond the fair use provisions granted by the U.S. Copyright Law is authorized by SPIE subject to payment of copying fees. The Transactional Reporting Service base fee for this volume is $\$ 18.00$ per article (or portion thereof), which should be paid directly to the Copyright Clearance Center (CCC), 222 Rosewood Drive, Danvers, MA 01923. Payment may also be made electronically through CCC Online at copyright.com. Other copying for republication, resale, advertising or promotion, or any form of systematic or multiple reproduction of any material in this book is prohibited except with permission in writing from the publisher. The CCC fee code is 0277-786X/09/\$18.00.

Printed in the United States of America.

Publication of record for individual papers is online in the SPIE Digital Library.

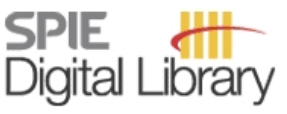

SPIEDigitalLibrary.org

Paper Numbering: Proceedings of SPIE follow an e-First publication model, with papers published first online and then in print and on CD-ROM. Papers are published as they are submitted and meet publication criteria. A unique, consistent, permanent citation identifier (CID) number is assigned to each article at the time of the first publication. Utilization of CIDs allows articles to be fully citable as soon they are published online, and connects the same identifier to all online, print, and electronic versions of the publication. SPIE uses a six-digit CID article numbering system in which:

- The first four digits correspond to the SPIE volume number.

- The last two digits indicate publication order within the volume using a Base 36 numbering system employing both numerals and letters. These two-number sets start with 00, 01, 02, 03, 04 , $05,06,07,08,09,0 A, 0 B \ldots$. OZ, followed by 10-1Z, 20-2Z, etc.

The CID number appears on each page of the manuscript. The complete citation is used on the first page, and an abbreviated version on subsequent pages. Numbers in the index correspond to the last two digits of the six-digit CID number. 


\section{Contents}

ix Conference Committee

\section{SESSION 1 HIGH POWER LASER SOURCES}

719802 High-brightness kW QCW diode laser stacks with ultra-low pitches [7198-01]

D. Schleuning, R. Pathak, C. Luong, E. Weiss, T. Hasenberg, Coherent, Inc. (United States)

719803 Brilliant low-fill-factor diode laser bars at 9xx $\mathrm{nm}$ for fiber coupling [7198-02]

H. König, G. Grönninger, C. Laver, A. Hammer, J. Maric, U. Strauß, OSRAM Opto

Semiconductors GmbH (Germany); H. Kissel, M. Haag, J. Biesenbach, DILAS Diodenlaser GmbH (Germany)

719804 Power scaling of bars toward $85 \mathrm{~mW}$ per $1 \mu \mathrm{m}$ stripe width reliable output power [7198-03] M. Krejci, Y. Gilbert, J. Müller, R. Todt, S. Weiss, N. Lichtenstein, Bookham AG (Switzerland)

719805 Continuous improvement of high-efficiency high-power 800-980nm diode lasers at Spectra-Physics [7198-04]

H. Li, T. Towe, I. Chyr, X. Jin, R. Miller, O. Romero, D. Liu, D. Brown, T. Truchan, T. Nguyen,

T. Crum, E. Wolak, R. Bullock, J. Mott, J. Harrison, Newport Spectra-Physics (United States)

$719806 \quad 640-\mathrm{nm}$ laser diode for small laser display [7198-05]

N. Shimada, M. Yukawa, K. Shibata, K. Ono, T. Yagi, A. Shima, Mitsubishi Electric Corp. (Japan)

719807 Progress in ultra-compact green frequency doubled optically pumped surface emitting lasers [7198-06]

U. Steegmueller, OSRAM Opto Semiconductors Inc. (United States); M. Kuehnelt, H. Unold, T. Schwarz, M. Schmitt, K. Aven, R. Schulz, C. Walter, I. Pietzonka, S. Illek, H. Lindberg,

A. Gomez-Iglesias, M. Furitsch, C. Laver, U. Strauss, T. Hoefer, OSRAM Opto Semiconductors GmbH (Germany)

$719808915 \mathrm{~nm}$ laser bar-based high-performance sources for fiber laser pumping [7198-07] R. Pathak, J. Minelly, J. Haapamaa, J. Watson, D. Schleuning, H. Winhold, E. Weiss, Coherent, Inc. (United States); M. Toivonen, S. Lehkonen, Coherent, Inc. (Finland); D. Roh, N. Ostrom, D. Grasso, J. Pfaff, T. C. Hasenberg, Coherent, Inc. (United States)

719809 Improved laser diode for high power and high temperature applications [7198-08] D. Schröder, M. Schröder, E. Werner, J. Meusel, D. Lorenzen, P. Hennig, JENOPTIK Laserdiode GmbH (Germany); R. Hülsewede, J. Sebastian, JENOPTIK Diode Lab. GmbH (Germany)

7198 OA Optimized high-power diode laser, laser arrays, and bars for pump applications [7198-09] R. Hülsewede, H. Schulze, J. Sebastian, JENOPTIK Diode Lab. GmbH (Germany); D. Schröder, J. Meusel, J. Wolf, P. Hennig, JENOPTIK Laserdiode GmbH (Germany) 
$7198 \mathrm{OB} \quad 5 \mathrm{~mm}$ long broad-area lasers at $976 \mathrm{~nm}$ with $\mathbf{6 5 \%}$ wall-plug efficiency [7198-10]

J. Gilly, P. Friedmann, m2k-laser GmbH (Germany); H. Kissel, J. Biesenbach, DILAS

Diodenlaser GmbH (Germany); M. T. Kelemen, m2k-laser GmbH (Germany)

\section{SESSION $2 \quad$ HIGH POWER DIODE LASER PACKAGING}

7198 OC Space-grade reliability of $808 \mathrm{~nm}$ QCW laser diode arrays (LDAs) delivering over 20 billion shots [7198-49]

Y. Berk, Y. Karni, G. Klumel, M. Levy, Y. Openhaim, S. Risemberg, SCD - Semi Conductor Devices (Israel); M. Rech, H. Becht, Carl Zeiss Optronics GmbH (Germany); B. Frei, F. Monti di Sopra, LASAG AG (Switzerland)

7198 OD Quasi-passive heat sink for high-power laser diodes [7198-12]

J. Vetrovec, Aqwest LLC (United States)

7198 OE High-power operation of $1 \mathrm{~cm}$ laser diode bars on funryu heat sink cooled by fluorinated-refrigerant [7198-13]

S. Oishi, H. Miyajima, N. Suzuki, T. Natsume, T. Fujita, K. Nishida, H. Kurino, R. Okamoto,

N. Kageyama, N. Suzuki, T. Uchiyama, H. Kan, Hamamatsu Photonics K.K. (Japan)

7198 OF High-power IR laser in SMT package [7198-57]

B. Pritsch, M. Behringer, M. Arzberger, C. Wiesner, R. Fehse, J. Heerlein, J. Maric, W. Giziewicz, OSRAM Opto Semiconductors GmbH (Germany)

$71980 \mathrm{~A}$ Automated assembly of fast-axis collimation (FAC) lenses for diode laser bar modules [7198-15]

J. Miesner, ficonTEC GmbH (Germany); A. Timmermann, J. Meinschien, LIMO Lissotschenko Mikrooptik GmbH (Germany); B. Neumann, S. Wright, Artifex Engineering e.K. (Germany);

T. Tekin, H. Schröder, Fraunhofer Institute for Reliability and Microintegration IZM (Germany); T. Westphalen, Fraunhofer Institute for Laser Technology (Germany); F. Frischkorn, ficonTEC GmbH (Germany)

$7198 \mathrm{OH} \quad$ Investigation of $n$-side cooling in regards to bar geometry and packaging style of diode laser [7198-16]

M. Leers, T. Westphalen, Fraunhofer Institute for Laser Technology (Germany); R. Pathak, C. Scholz, Coherent Inc. (United States)

71980 Ol CTE-matched microchannel coolers [7198-17]

G. Rudd, K. Kusnadi, R. Brox, Spectra-Mat, Inc. (United States); A. Grantz, Grantz Engineering (United States)

$71980 \mathrm{~J} \quad$ Long-term wavelength stability of high-power laser diode bars on microchannel coolers [7198-18]

D. R. Balsley, D. C. Dawson, R. Johnson, R. J. Martinsen, nLight Corp. (United States)

7198 OK Packaging influence on laser bars of different dimensions [7198-19]

T. Westphalen, M. Leers, M. Werner, M. Traub, H.-D. Hoffmann, Fraunhofer Institute for Laser Technology (Germany); R. Ostendorf, Fraunhofer Institute for Applied Solid State Physics (Germany) 
$71980 \mathrm{M} \quad$ Applications of high-power diode lasers for aluminum welding [7198-21]

S. Huber, Technische Univ. München (Germany); M. Merzkirch, Univ. Karlsruhe (Germany); M. F. Zaeh, Technische Univ. München (Germany); V. Schulze, Univ. Karlsruhe (Germany)

7198 ON Improvements of high-power diode laser line generators open up new application fields [7198-22]

J. Meinschien, A. Bayer, P. Bruns, L. Aschke, V. N. Lissotschenko, LIMO Lissotschenko Mikrooptik GmbH (Germany)

\section{SESSION 4 HIGH POWER FIBER COUPLED SOURCES I}

$719800 \quad$ High-brightness fiber coupled pumps [7198-24]

V. Gapontsev, N. Moshegov, P. Trubenko, A. Komissarov, I. Berishev, O. Raisky, N. Strougov, V. Chuyanov, G. Kuang, O. Maksimov, A. Ovtchinnikov, IPG Photonics Corp. (United States)

7198 OP $\quad 100 \mathrm{~W} / 100 \mu \mathrm{m}$ passively cooled fiber coupled diode laser at $976 \mathrm{~nm}$ based on multiple 100 $\mu \mathrm{m}$ single emitters [7198-25]

M. Werner, C. Wessling, S. Hengesbach, M. Traub, H.-D. Hoffmann, Fraunhofer Institute for Laser Technology (Germany)

$71980 Q \quad$ High-brightness fiber-coupled single emitter arrays [7198-26]

S. Heinemann, B. Regaard, T. Schmidt, B. Lewis, Fraunhofer USA Inc. (United States)

7198 OR High-power fiber-coupled modules based on tapered diode lasers at $975 \mathrm{~nm}$ [7198-27] G. Kochem, M. Traub, S. Hengesbach, D. Hoffmann, Fraunhofer Institute for Laser Technology (Germany)

7198 OS Recent progress in fiber-coupled multi-mode pump module and broad-area laser-diode performance from $800-1500 \mathrm{~nm}$ [7198-28]

V. Wong, V. Rossin, J. Skidmore, P. Yalamanchili, X. Qui, R. Duesterberg, P. Doussiere,

D. Venables, R. Raju, J. Guo, M. Au, L. Zavala, M. Peters, G. Yang, Y. Dai, E. Zucker, JDSU (United States)

7198 OT $\quad$ 100-W 105- 1 m 0.15NA fiber coupled laser diode module [7198-29]

S. R. Karlsen, R. K. Price, M. Reynolds, A. Brown, R. Mehl, S. Patterson, R. J. Martinsen, nLight Corp. (United States)

7198 OU High-brightness fiber-coupled pump sources at $88 \mathrm{X} \mathbf{n m}$ and $79 \mathrm{X} \mathbf{n m}$ [7198-30] J. Watson, D. Schleuning, H. Winhold, M. Griffin, S. Tolman, R. Pathak, E. Weiss, T. Hasenberg, Coherent, Inc. (United States)

7198 OV Advances in fiber combined pump modules for fiber lasers [7198-31] T. Crum, O. Romero, H. Li, X. Jin, T. Towe, I. Chyr, T. Truchan, D. Liu, S. Cutillas, K. Johnson, S.-K. Park, E. Wolak, R. Miller, R. Bullock, J. Mott, B. Fidric, J. Harrison, Newport Spectra-Physics (United States)

7198 OW High-peak-power fiber coupled super-pulsed diode lasers [7198-32] M. Traub, M. Giesberts, J. Geiger, C. Johnigk, H.-D. Hoffmann, Fraunhofer Institute for Laser Technology (Germany) 
$71980 X \quad$ Intensity increasing up to $4 \mathrm{MW} / \mathrm{cm} 2$ with BALB's via wavelengths coupling [7198-33] A. Timmermann, D. Bartoschewski, S. Schlüter, C. Burke, J. Meinschien, LIMO Lissotschenko Mikrooptik GmbH (Germany)

\section{SESSION 5 HIGH POWER FIBER COUPLED SOURCES II}

7198 OY Very high-brightness fiber-coupled diode lasers [7198-34]

S. D. Roh, D. M. Grasso, J. A. Small, Coherent, Inc. (United States)

$71980 Z$ A high power high-brightness multi-single-emitter laser pump platform [7198-35]

X. Qiu, Y. Dai, M. Au, J. Guo, V. Wong, V. Rossin, D. Venables, J. Skidmore, E. Zucker, JDSU (United States)

\section{SESSION 6 HIGH BRIGHTNESS LASER SOURCES}

719810 Wavelength stabilized high-power diode laser modules [7198-36]

B. Köhler, T. Brand, M. Haag, J. Biesenbach, DILAS Diodenlaser GmbH (Germany)

$719811 \quad$ High-power frequency stabilized tapered diode amplifiers at $1064 \mathrm{~nm}$ [7198-37]

R. Ostendorf, C. Schilling, G. Kaufel, R. Moritz, J. Wagner, Fraunhofer Institute for Applied Solid State Physics (Germany); G. Kochem, Fraunhofer Institute for Laser Technology (Germany); P. Friedmann, J. Gilly, M. T. Kelemen, m2k-laser GmbH (Germany)

719812 High-performance wavelength-locked diode lasers [7198-38]

P. Leisher, K. Price, S. Karlsen, D. Balsley, D. Newman, R. Martinsen, S. Patterson, nLight Corp. (United States)

\section{SESSION 7 RELIABILITY AND FAILURE ANALYSIS}

$71981420 \mathrm{~W}$ continuous wave reliable operation of $980 \mathrm{~nm}$ broad-area single emitter diode lasers with an aperture of 96 $\mu \mathrm{m}$ [7198-40]

P. Crump, G. Blume, K. Paschke, R. Staske, A. Pietrzak, U. Zeimer, S. Einfeldt, A. Ginolas, F. Bugge, K. Häusler, P. Ressel, H. Wenzel, G. Erbert, Ferdinand-Braun-Institut für Höchstfrequenztechnik (Germany)

719815 Fault protection of broad-area laser diodes [7198-41]

J. H. Jacob, R. Petr, M. A. Jaspan, S. D. Swartz, M. T. Knapczyk, A. M. Flusberg, Science Research Lab., Inc. (United States); A. K. Chin, Aland Chin, LLC (United States); I. Smilanski, Science Research Lab., Inc. (United States)

719816 Reliability of diode lasers for space applications [7198-42]

K. Häusler, U. Zeimer, B. Sumpf, F. Bugge, P. Ressel, G. Erbert, G. Tränkle,

Ferdinand-Braun-Institut für Höchstfrequenztechnik (Germany)

719817 Improved brightness on broad-area single emitter (BASE) modules [7198-43]

S. Pawlik, A. Guarino, N. Matuschek, R. Bättig, S. Arlt, Bookham AG (Switzerland); D. Lu, Bookham Technology Co., Ltd. (China); N. Zayer, J. Greatrex, Bookham Technology, plc (United Kingdom); B. Sverdlov, B. Valk, N. Lichtenstein, Bookham AG (Switzerland) 
719818 Catastrophic facet and bulk degradation in high power multi-mode InGaAs strained quantum well single emitters [7198-44]

Y. Sin, N. Presser, B. Foran, N. Ives, S. C. Moss, The Aerospace Corp. (United States)

719819 Reliability of single-mode and multi-mode high-power semiconductor lasers at eye-safe wavelengths [7198-45]

T. Stakelon, J. Lucas, M. Osowski, R. Lammert, S. Moon, C. Panja, V. Elarde, K. Gallup, W. Hu, J. Ungar, QPC Lasers, Inc. (United States)

$71981 \mathrm{~A}$ A comprehensive model of catastrophic optical-damage in broad-area laser diodes [7198-46]

A. K. Chin, Aland Chin, LLC (United States); R. K. Bertaska, New England Analytical, LLC (United States); M. A. Jaspan, A. M. Flusberg, S. D. Swartz, M. T. Knapczyk, R. Petr, I. Smilanski, J. H. Jacob, Science Research Lab., Inc. (United States)

\section{POSTER SESSION}

7198 1C Laser applied on railway line profile measurement [7198-23]

C. Peng, L. Wang, Z. Wang, X. Gao, K. Yang, J. Peng, Y. Zhang, Southwest Jiaotong Univ. (China)

7198 1D Reaching 1 watt reliable output power on single-mode $980 \mathbf{n m}$ pump lasers [7198-48] M. Bettiati, V. Cargemel, P. Pagnod, C. Hervo, P. Garabedian, P. Issert, L. Raymond, L. Ragot, J.-C. Bertreux, J.-N. Reygrobellet, C. Crusson, F. Laruelle, 3S PHOTONICS SA (France)

7198 1E High reliability operation of 2 kW QCW 10-bar laser diode stacks at 808 nm [7198-50] V. Loyo-Maldonado, G. Bacchin, S. Robertson, K. Man, B. Qiu, S. D. McDougall, J. H. Marsh, Intense Ltd. (United Kingdom)

7198 IF High-power QCW arrays for operation over wide temperature extremes [7198-51] R. Feeler, J. Junghans, E. Stephens, Northrop Grumman Cutting Edge Optronics (United States)

$71981 \mathrm{G}$ Assessment of the limits to peak power of $1100 \mathrm{~nm}$ broad area single emitter diode lasers under short pulse conditions [7198-52]

X. Wang, P. Crump, A. Pietrzak, C. Schultz, A. Klehr, T. Hoffmann, A. Liero, A. Ginolas, S. Einfeldt, F. Bugge, G. Erbert, G. Tränkle, Ferdinand-Braun-Institut für Höchstfrequenztechnik (Germany)

$7198 \mathrm{1H} \quad$ High wall-plug efficiency diode lasers with an Al-free active region at $975 \mathrm{~nm}$ [7198-53] N. Michel, M. Calligaro, Y. Robert, M. Lecomte, O. Parillaud, M. Krakowski, Alcatel-Thales III-V Lab. (France); T. Westphalen, M. Traub, Fraunhofer-Institut für Lasertechnik (Germany)

$719811 \quad$ High d/gamma values in diode laser structures for very high power [7198-54] I. B. Petrescu-Prahova, P. Modak, E. Goutain, D. Silan, D. Bambrick, J. Riordan, T. Moritz, Intense US (United States); S. D. McDougall, B. Qiu, J. H. Marsh, Intense UK (United Kingdom)

$71981 \mathrm{~J} \quad$ Power scaling of mini-bar-based high-power 780-980nm pump modules at Spectra-Physics [7198-55]

D. Liu, S. Cutillas, X. Jin, S.-K. Park, K. Johnson, H. Li, T. Truchan, E. Wolak, T. Towe, I. Chyr, R. Miller, A. Meissner, D. Lenarduzzi, R. Bullock, J. Mott, J. Harrison, Newport Spectra-Physics (United States) 
7198 1K Material survey for packaging semiconductor diode lasers [7198-56]

D. Schleuning, K. Scholz, M. Griffin, B. Guo, C. Luong, R. Pathak, C. Scholz, J. Watson,

H. Winhold, T. Hasenberg, Coherent, Inc. (United States)

$71981 \mathrm{~L} \quad$ Wavelength-stabilized tapered laser diodes in an external Talbot cavity: simulations and experiments [7198-59]

D. Paboeuf, G. Lucas-Leclin, P. Georges, Lab. Charles Fabry de L'Institut d'Optique, CNRS, Univ Paris-Sud (France); N. Michel, M. Krakowski, Alcatel-Thales III-V Lab. (France); J. J. Lim, S. Sujecki, E. Larkins, Univ. of Nottingham (United Kingdom)

7198 1N High-power semiconductor lasers for applications requiring GHz linewidth source [7198-61] I. Divliansky, The College of Optics and Photonics, Univ. of Central Florida (United States); V. Smirnov, OptiGrate (United States); G. Venus, A. Gourevitch, L. Glebov, The College of Optics and Photonics, Univ. of Central Florida (United States)

719810 Wheel profile dynamic inspection system based on slit diode laser [7198-62] Y. Zhang, J. Peng, L. Wang, X. Gao, Z. Wang, Q. Zhao, C. Peng, K. Yang, Southwest Jiaotong Univ. (China)

7198 1P Dynamic detection for the contact line gradient in electrified railway [7198-63] J. Peng, Y. Zhang, L. Wang, X. Gao, Z. Wang, Q. Zhao, C. Peng, K. Yang, Southwest Jiaotong Univ. (China)

7198 IR High-spectral brightness pump sources for diode-pumped solid state lasers [7198-65] W. Hu, QPC Lasers, Inc. (United States); F. D. Patel, Physical Optics Corp. (United States); M. L. Osowski, R. M. Lammert, S. W. Oh, QPC Lasers, Inc. (United States); C. Panja, Ostendo Technologies (United States); V. C. Elarde, L. Vaissié, J. E. Ungar, QPC Lasers, Inc. (United States)

Author Index 


\title{
Conference Committee
}

\author{
Symposium Chairs \\ Donald J. Harter, IMRA America, Inc. (United States) \\ Peter R. Herman, University of Toronto (Canada) \\ Symposium Cochairs
}

Henry Helvajian, The Aerospace Corporation (United States)

Friedrich G. Bachmann, Rofin-Sinar Laser GmbH (Germany)

Program Track Chair

E. Fred Schubert, Rensselaer Polytechnic Institute (United States)

Conference Chair

Mark S. Zediker, Consultant (United States)

Program Committee

Friedrich G. Bachmann, Rofin-Sinar Laser GmbH (Germany)

Stefan W. Heinemann, Fraunhofer USA Inc. (United States)

Volker K. Krause, Laserline GmbH (Germany)

Robert J. Martinsen, nLight Corporation (United States)

Erik P. Zucker, JDSU (United States)

Session Chairs

$1 \quad$ High Power Laser Sources

Robert J. Martinsen, nLight Corporation (United States)

2 High Power Diode Laser Packaging

Stefan W. Heinemann, Fraunhofer USA Inc. (United States)

3 Laser Diode Applications

Friedrich G. Bachmann, Rofin-Sinar Laser GmbH (Germany)

$4 \quad$ High Power Fiber Coupled Sources I

Erik P. Zucker, JDSU (United States)

$5 \quad$ High Power Fiber Coupled Sources II

Erik P. Zucker, JDSU (United States) 
$6 \quad$ High Brightness Laser Sources

Mark S. Zediker, Consultant (United States)

$7 \quad$ Reliability and Failure Analysis

Volker K. Krause, Laserline GmbH (Germany) 